\title{
The food and nutrient intake of 5- to 12-year-old Australian children during school hours: a secondary analysis of the 2011-2012 National Nutrition and Physical Activity Survey
}

\author{
Alexandra C Manson ${ }^{1}$ (D), Brittany J Johnson ${ }^{1, *}{ }^{(0)}$, Dorota Zarnowiecki ${ }^{1}$ (ㅇ, \\ Rachel Sutherland ${ }^{2,3,4,5}$ and Rebecca K Golley ${ }^{1}$ (i) \\ ${ }^{1}$ Caring Futures Institute, College of Nursing and Health Sciences, Flinders University, Bedford Park, GPO Box 2100 , \\ Adelaide, SA 5001, Australia: ${ }^{2}$ Hunter New England Population Health, Wallsend, NSW, Australia: ${ }^{3}$ School of \\ Medicine and Public Health, University of Newcastle, Callaghan, NSW, Australia: ${ }^{4}$ Hunter Medical Research Institute, \\ New Lambton Heights, NSW, Australia: ${ }^{5}$ Priority Research Centre for Health Behaviour, School of Medicine and Public \\ Health, University of Newcastle, Callaghan, NSW, Australia
}

Submitted 29 March 2021: Final revision received 27 August 2021: Accepted 3 September 2021: First published online 8 September 2021

\begin{abstract}
Objective: School food intake of Australian children is not comprehensively described in literature, with limited temporal, nationally representative data. Greater understanding of intake at school can inform school-based nutrition promotion. This study aimed to describe the dietary intake of primary-aged children during school hours and its contribution to daily intake.

Design: This secondary analysis used nationally representative, cross-sectional data from the 2011 to 2012 National Nutrition and Physical Activity Survey. Dietary intake was assessed using validated 24-h dietary recalls on school days. Descriptive statistics were undertaken to determine energy, nutrients, food groups and food products consumed during school hours, as well as their contributions to total daily intake. Associations between school food intake and socio-demographic characteristics were explored.

Setting: Australia.

Participants: Seven hundred and ninety-five children aged 5-12 years.

Results: Children consumed $37 \%$ of their daily energy and 31-43\% of select nutrient intake during school hours, with discretionary choices contributing $44 \%$ of school energy intake. Most children consumed less than one serve of vegetables, meat and alternatives or milk and alternatives during school hours. Commonly consumed products were discretionary choices (34\%, including biscuits, processed meat), bread (17\%) and fruit (12\%). There were limited associations with socio-economic position variables, apart from child age.

Conclusions: Children's diets were not aligned with national recommendations, with school food characterised by high intake of discretionary choices. These findings are consistent with previous Australian evidence and support transformation of the Australian school food system to better align school food consumption with recommendations.
\end{abstract}

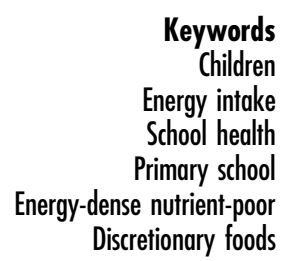

Children

Energy intake

School health

Dutrient-poor Discretionary foods
Children's dietary habits during childhood are linked to risk of developing chronic conditions and food consumption patterns in adulthood ${ }^{(1)}$. Within Australia, children's diets are profiled by a high intake of discretionary food products that is, energy-dense, nutrient-poor foods higher in energy, saturated fat, added sugars and/or $\mathrm{Na}$; with these foods contributing over a third (38.5\%) of children's and adolescent's daily energy intake ${ }^{(1,2)}$. Concurrently, children are not meeting national recommendations for vegetables, fruit and milk foods and alternatives ${ }^{(3)}$. Similar dietary trends are observed in children internationally ${ }^{(4)}$, with greater than $91 \%$ of children in the USA exceeding recommendations for solid fats and added sugars ${ }^{(5)}$.

Schools are a key environment for health promotion and nutrition interventions, providing an opportunity to align children's diets with recommendations ${ }^{(6)}$. Children spend 
6-7 h in school daily ${ }^{(7)}$, with international evidence showing food consumed during school hours contributes between $24 \%$ and $44 \%$ of daily energy intake ${ }^{(4,8,9)}$. Internationally, there are three predominant school food provision models including: (1) meals provided by the school (e.g. in a cafeteria format); (2) food brought to school from home (e.g. in a 'lunchbox') or (3) a combination of these models. Past analyses of school provided models have found children are often supplied with foods that make up $23 \%$ to $33 \%$ of their daily energy intake $(8,10,11)$ in the form of cooked meals, including vegetables ${ }^{(12-14)}$. However, school provided meals have been reported to include several energy-dense, nutrient-poor foods, such as baked sweets or hot chips ${ }^{(12)}$. Contrastingly, examination of lunchbox models internationally has found lunchboxes provide $33 \%$ to $44 \%$ of children's daily energy intake $^{(4,9,10,15)}$ and Australian evidence showing $37 \%$ contribution of lunchbox energy to overall daily energy intake $^{(16)}$. Lunchboxes commonly include fruit, breads, discretionary choices, such as packaged snacks or processed meats, and limited vegetables ${ }^{(10,12)}$

In Australia, on any given day, $86 \%$ of primary schoolaged children consume foods brought in a lunchbox from home with a small proportion of children purchasing lunch or snacks from a school canteen facility ${ }^{(16)}$ (i.e. a small shop within the school where students can purchase lunch, snacks and beverages $)^{(17)}$. While there are national dietary guidelines $^{(1)}$, providing food recommendations for children across the day, and international guidelines to support the provision of healthy food at school consistent with dietary guidelines $^{(18)}$, there are no national lunchbox policies to mandate food provision during school by caregivers. National canteen recommendations are available to guide the nutritional quality of food options served at canteens, supported by state/territory policies ${ }^{(19)}$. Regardless of existing guidelines, there are several barriers for school food provision by both caregivers and canteens, including food safety, facilities available, time, preference and cost ${ }^{(20)}$.

Research into Australian children's school food intake is limited in terms of national, temporal evidence, collected using validated measures of intake. The latest analysis of school food intake using a nationally representative sample is outdated, using data collected in $1995^{(16)}$. Since this previous data collection, there have been changes to school food policies and practices, including the national dissemination of fruit and vegetable snack time 'Crunch and Sip' ${ }^{\text {(21) }}$ and national canteen guidelines ${ }^{(19)}$. Therefore, findings may no longer reflect the dietary intake of Australian primary-aged population, hence analysis using more contemporary data is required. More recent findings from a sample of 12 catholic schools in the Hunter region of New South Wales $^{(22)}$ found that $67 \%$ of lunchboxes contained sweet discretionary snacks (e.g. sweet biscuits, cakes, muesli bars), and $55 \%$ contained savoury discretionary snacks (e.g. crisps, savoury biscuits). While this study used objective direct observation, it measured provision of foods in lunchboxes, rather than intake, and lacked comparison to total day intake. Furthermore, previous studies have involved primary school children from selected states or regions, therefore do not provide a national perspective of children's intake ${ }^{(22-24)}$. More broadly, available descriptions of Australian children's lunchboxes lack comprehensive analysis of nutrient content and discretionary food contribution $^{(22)}$ necessary to assess and guide intervention development.

A thorough understanding of Australian children's school food intake will allow for better informed public health and nutrition promotion strategies to be developed. Hence, this study aimed to describe the dietary intake of 5- to 12-year-old (primary-aged) Australian children during school hours and the contribution of this school day intake to their total day intake, using a nationally representative sample. Specifically, we sought to: (1) determine the energy and nutrients from core and discretionary choices consumed during school hours, as well as their contributions to total daily intake; (2) examine the consumption of Australian Dietary Guidelines food groups within school hours; (3) identify the types of discretionary choices most consumed during school hours and (4) identify associations between child socio-demographic characteristics and percentage of energy from discretionary choices consumed during school hours.

\section{Methods}

\section{Data source}

This secondary analysis was completed using crosssectional data collected by the National Nutrition and Physical Activity Survey (NNPAS) 2011-2012(25). Ethics approval was not required for the secondary analysis as all data provided were deidentified. Detailed methods of the NNPAS 2011-2012 can be found in the Australian Health Survey User Guide ${ }^{(25)}$. In brief, participants were recruited through a stratified multistage area sample of private households, interviewing an adult and child, if present ${ }^{(25)}$. Dietary intake data were collected by $24-\mathrm{h}$ recalls on two separate days of intake using the Automated Multiple Pass Method ${ }^{(26)}$ between May 2011 to June $2012^{(25)}$. The first day of dietary recalls were performed face to face with $64 \%$ of the sample completing the second recall over the phone. All recalls were conducted with a parent proxy for children under the age of 15 years, with the child assisting where appropriate ${ }^{(25)}$.

\section{Dataset preparation}

Only day one recalls, completed on a weekday, by participants aged between 5 and 12 years of age were included in this analysis (Fig. 1). Recalls completed on dates that were holidays across all Australian states (except Tasmania) and territories were excluded, forming the primary sample. 
The dietary intake of children during school

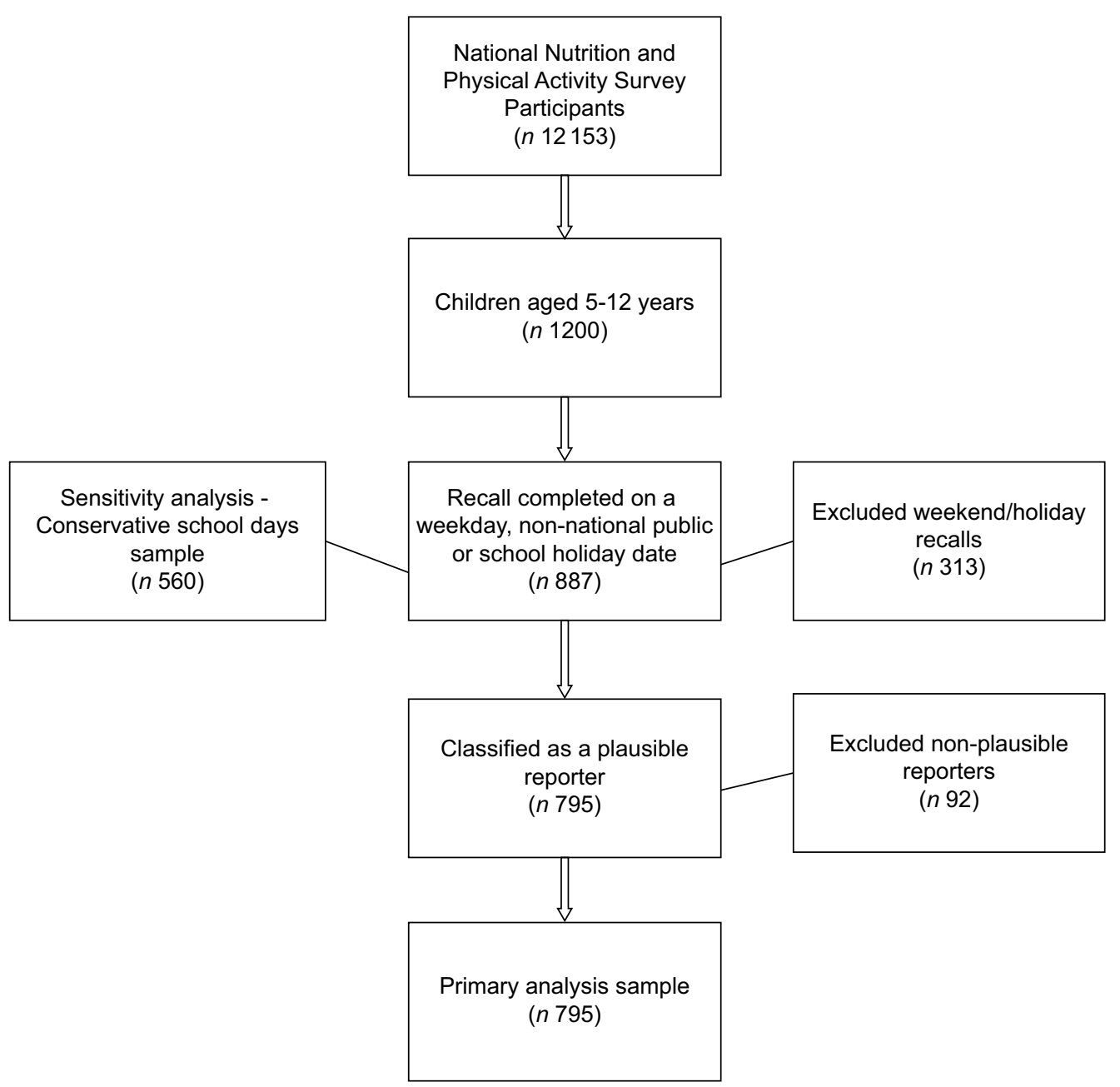

Fig. 1 Participant flow of the sample of 5- to 12-year-old children from the NNPAS 2011-2012*. *National Nutrition and Physical activity survey $2011-2012$

State or territory of residence information was not available in the dataset, therefore dates that were national public holidays or school holidays in 2011 and 2012 across most states/territories nationally were excluded. School term dates for Tasmania were not considered due to the state following a trimester school term structure; however, participant data were still included. School food was defined as food consumed between 9 am and $3 \mathrm{pm}$, as these are common national school hours and broadly capture all school eating occasions, including morning snack, recess and lunch times. To account for potential over and under reporting in the 24-h diet recall, only plausible reporters were included. Plausible reporters were defined using the Goldberg cut-off method ${ }^{(27,28)}$, which compares energy intake (EI) and BMR ratio with estimated energy expenditure (physical activity level, PAL). This utilised the EI: BMR variables provided in the dataset, calculated using diet recall and anthropometric information. This ratio, with the addition of PAL, provides an indication if the energy intake meets energy requirements for standard energy expenditure. Physical activity level was assumed to be
1.55 for all reporters due to lack of consistent physical activity measures available in this dataset. Plausible reporters were determined as those within two standard deviations of a PAL of $1.55(0.87$ to 2.74$)$. Participants without anthropometric data ( $n$ 162, $17 \%$ ) were included as plausible reporters as the plausibility of their reported intake was unable to be assessed and therefore assumed to be plausible to maintain a larger primary analysis sample $\mathrm{e}^{(29)}$.

\section{Dietary intake data}

The primary dependent variables included energy and select nutrient intake, food group serves and commonly consumed food products, with the independent variables being school hours and contribution to the total day. The Australian Food, Supplement and Nutrient Database (AUSNUT) 2011-2013 ${ }^{(25)}$, developed by Food Standards Australia and New Zealand based on food products consumed in the NNPAS 2011-2012 ${ }^{(30)}$, was used to analyse food composition data. The nutrient profiles of food consumed during school hours and across the total day were 
collated into separate variables, as primary variables. Secondary variables included the contribution of discretionary choices to energy and nutrients consumed during school hours.

Food groups were defined according to Australian Dietary Guidelines, which categorises foods and beverages into five food groups and discretionary choices, and includes recommended daily serves of food groups, based on age and $\operatorname{sex}^{(1,25)}$. The five food groups are: (1) vegetables and legumes/beans; (2) fruit; (3) grain (cereal) foods, mostly wholegrain and/or high cereal fibre varieties; (4) lean meats and poultry, fish, eggs, tofu, nuts and seeds and legumes/beans; and (5) milk, yoghurt, cheese and/ or alternatives, mostly reduced fat ${ }^{(1)}$. The guidelines also categorise foods not required for a healthy diet which are high in energy, saturated fat, added sugars and/or $\mathrm{Na}$ and low in nutrients as discretionary choices and recommends minimising intake of these foods ${ }^{(1)}$. Serves of each of the five food groups and discretionary choices were calculated for all items consumed, using the grams consumed and serve size definitions ${ }^{(1)}$. This was collated into total serves consumed and those consumed during school hours. This allowed primary variables, including serves consumed during school hours, and secondary variables, including total serves consumed, to be determined. Food products were also categorised to determine food type by the food category and subcategories defined in the AUSNUT 2013 food composition dataset ${ }^{(30)}$.

\section{Covariates}

Characteristics of participants were available in the NNPAS 2011-2012 dataset. Anthropometric measures for weight and height were taken by interviewers using standard procedures and used to calculate $\mathrm{BMI}^{(25)}$. BMI was provided in the dataset, calculated using age- and sex-specific cut-off points sourced from Cole et $a l^{(31)}$, and used to determine weight status categories of participants. Data for sociodemographic characteristics were collected during the interview, including age in whole years, sex, household income and postcode. Household income in the NNPAS dataset was transformed into "equivalised household income," adjusting for household size and separated into deciles $^{(25)}$. Socio-economic position was classified using the Socio-economic Indexes for Areas (SEIFA) Index of Relative Socio-Economic Disadvantage (2011), derived from household postcode and measured in quintiles; this variable was pre-calculated in the available version of the dataset ${ }^{(25)}$. This index considers income, education and employment in certain living areas, showing a measure of social and economic well-being in that region, with lower quintiles representing areas with more disadvantage $^{(25)}$. Additionally, physical activity of participants was measured through the number of days in the past week that $1 \mathrm{~h}$ of physical activity was completed.

\section{Statistical analysis}

All statistical analyses were performed in IBM SPSS Statistics 25 (version 25; SPSS Inc.). Normality assessment, using histograms and skewness and kurtosis $Z$-scores, showed data were positively skewed, therefore median and interquartile range (IQR) were examined. Descriptive statistics were used to determine energy and select nutrient intake during school hours and the total day. The percentage contribution of school energy intake to total intake and discretionary choice contribution to school intake were calculated for each participant and therefore mean percentage intake for the sample was determined. This allowed for comparison of the nutrients consumed in school hours and the contribution of these to the total day. Intake of food groups were calculated using median serves in school hours and total day. The most frequently consumed discretionary food and beverage products across the sample during school hours were calculated, to identify most frequently consumed types of this diverse food group.

Multivariate linear regression analyses were used to determine the relationship between child sociodemographic characteristics and percentage of energy from discretionary choices during school hours. The predictor variables were assessed for collinearity with no associations found. Multivariate regressions were run ( $n$ 627) including participants with complete weight, age and socio-demographic data available. Model one included socio-demographic characteristics of interest and biological factors which are known to effect energy requirements ${ }^{(32)}$. This included the independent variables of SEIFA, household income deciles, child weight status and child age. Model two included all variables for model one, and additionally controlled for child sex and physical activity level (determined from the number of days physical activity recommendations were met in a week), which have been associated with children's total energy intake in the literature ${ }^{(33)}$. Weight status categories were dummy-coded into new variables, with healthy weight coded as the reference category. SEIFA and income deciles, which were ordinal categories, were coded numerically and treated as continuous variables in the regression ${ }^{(34)}$. All other included variables were considered continuous in this analysis.

\section{Sensitivity analyses}

Sensitivity analyses were completed to understand whether defining school days using a more conservative method would influence the results. A subsample labelled as "conservative school days" was formed including only participants who completed dietary recalls on definite school days across all states and territories, forming a smaller sample ( $n$ 560). Sensitivity analyses were completed comparing the primary sample ( $n$ 795) to the sample of 
participants from conservative school days ( $n$ 560). This sensitivity analysis accounted for potential differences in the consumption patterns of the primary sample, which may have included some holiday dates, for example, for Tasmania, compared to the conservative sample, which included only recalls on confirmed school days.

\section{Results}

From the 1200 children aged between 5 and 12 years participating in the NNPAS 2011-2012, a total of 795 plausible reporters completed a dietary recall on a school day. Anthropometric and socio-demographic characteristics of the sample are presented in Table 1 . The mean age was 8.5 years (SD 2.3), with majority (69\%) of children being classified within a healthy weight category. Participants were distributed across all socio-economic quintiles.

Energy, nutrient and discretionary choices intake during school hours and contribution to total day

Table 1 Characteristics of the sample of 5- to 12-year-old children from the NNPAS 2011-12 reporting dietary intake on a school day $(n 795)$

\begin{tabular}{|c|c|c|}
\hline Characteristic & $n$ & $\%$ \\
\hline \multicolumn{3}{|l|}{ Sex } \\
\hline Male & 406 & 51.1 \\
\hline Female & 389 & 48.9 \\
\hline \multicolumn{3}{|l|}{ Child age (years) } \\
\hline Mean & 8.5 & \\
\hline SD & $2 \cdot 3$ & \\
\hline \multicolumn{3}{|c|}{ Socio-economic position* } \\
\hline Quintile 1 (lowest) & 141 & $17 \cdot 7$ \\
\hline Quintile 2 & 141 & $17 \cdot 7$ \\
\hline Quintile 3 & 158 & $19 \cdot 9$ \\
\hline Quintile 4 & 152 & $19 \cdot 1$ \\
\hline Quintile 5 (highest) & 203 & 25.5 \\
\hline \multicolumn{3}{|c|}{ Equivalised income of household $\dagger$} \\
\hline Decile 1 (lowest) & 91 & $12 \cdot 7$ \\
\hline Decile 2 & 41 & $5 \cdot 7$ \\
\hline Decile 3 & 75 & $10 \cdot 5$ \\
\hline Decile 4 & 74 & $10 \cdot 3$ \\
\hline Decile 5 & 87 & $12 \cdot 2$ \\
\hline Decile 6 & 78 & $10 \cdot 9$ \\
\hline Decile 7 & 87 & $12 \cdot 2$ \\
\hline Decile 8 & 75 & $10 \cdot 5$ \\
\hline Decile 9 & 55 & $7 \cdot 7$ \\
\hline Decile 10 (highest) & 53 & $7 \cdot 4$ \\
\hline \multicolumn{3}{|l|}{ Weight status $\ddagger$} \\
\hline Underweight & 38 & $6 \cdot 0$ \\
\hline Healthy weight & 433 & $69 \cdot 1$ \\
\hline Overweight & 111 & $17 \cdot 7$ \\
\hline Obesity & 45 & $7 \cdot 2$ \\
\hline \multicolumn{3}{|l|}{ Physical activity§ } \\
\hline None & 36 & 4.5 \\
\hline $1-2 d$ & 110 & $13 \cdot 8$ \\
\hline $3-5 d$ & 271 & $34 \cdot 1$ \\
\hline $6-7 d$ & 377 & 47.4 \\
\hline
\end{tabular}

*Measured by the socio-economic indexes for areas including the index of relative socio-economic disadvantage, quintiles.

$\dagger$ Equivalised by household size.

$\ddagger$ Missing weight data $n 162$. Weight status determined through age- and sexspecific BMI cut-off points.

$\S N u m b e r$ of days each child physical activity for at least $60 \mathrm{~min}$ in $7 \mathrm{~d}$ prior to interview.
Table 2 presents children's median energy ( $2578 \mathrm{~kJ}$, IQR 1925, 3595) and selected nutrient intake in school hours. Children consumed $37 \%$ of total day energy intake, and between $31 \%$ and $43 \%$ of their total day intake of nutrients in school hours (Table 2). Mean nutrient intake contribution of products consumed during school hours was lowest for protein (31\%), Fe (32\%), Ca (33\%), compared to highest for $\mathrm{Na}(43 \%)$, fibre ( $41 \%)$ and carbohydrate ( $40 \%)$. Of the food and beverages consumed during school hours, 43.8\% (Median $1033 \mathrm{~kJ}$, IQR 491, $1920 \mathrm{~kJ}$ ) of energy was from discretionary choices (see online Supplemental Table S1).

\section{Food group intake}

During school hours, children consumed a median of two serves of discretionary choices (IQR 1,3 ) and grains (IQR 1, 2) and one serve of fruit (IQR 0,1), and no serves from the meat and alternatives (IQR 0,0), milk foods and alternatives (IQR 0, 1) and vegetable food groups (IQR 0, 1) (Table 3). Across the total day, children consumed a median of five serves of discretionary choices (IQR 3, 7), four serves of grains (IQR 3, 6) and one serve of each of the vegetable (IQR 0, 3), fruit (IQR 1, 3), milk foods and alternatives (IQR 1, 2) and meat and alternatives (IQR 0, 2) food groups.

\section{Discretionary choices products most consumed during school bours}

During school hours, $82 \%$ of children consumed one or more serves of discretionary choices. Over a third (34\%) of the food products consumed during school hours were classified as discretionary choices, with other highly consumed foods including bread (17\%) and fruit (12\%). Of these discretionary choices, the most frequently consumed foods were sweet biscuits (14\%), processed meat (13\%), savoury biscuits (12\%) and muesli or cereal bars (10\%) (Fig. 2). The most frequently consumed beverages during school hours were water $(67 \%)$, juice $(19 \%)$, soft drink (6\%) and cordial (4\%).

\section{Associations between socio-demographic characteristics and percentage of energy from discretionary choices}

Multivariate linear regressions showed no significant associations $(P>0.05)$ between SEIFA Index (Standardised $\beta=-0.024, \quad P=0.571$ ), household income categories (Standardised $\beta=-0.060, P=0.148$ ) or weight status (Underweight $\quad$ Standardised $\quad \beta=0.001, \quad P=0.979$; Overweight Standardised $\beta=-0.034, P=0.418$; Obesity Standardised $\beta=-0.013, P=0.758)$ and percent of energy from discretionary choices during school hours, when adjusting for child sex and physical activity level $(F=(8585)=1.539, P=0 \cdot 140)$ (see online Supplemental Table S2). There was a significant association between age and percentage of discretionary energy consumed during school hours (Standardised $\beta=0.126, \quad P=0.003$ ), 
Table 2 Energy and nutrient intake of Australian 5- to 12-year-old children during school hours and across the total day using the NNPAS 2011-2012 ( $n$ 795)

\begin{tabular}{|c|c|c|c|c|c|}
\hline & \multicolumn{3}{|c|}{ In school hours } & \multicolumn{2}{|c|}{ Total day } \\
\hline & Median & IQR & $\begin{array}{c}\text { Mean } \% \text { contribution } \\
\text { to total day }{ }^{\star}\end{array}$ & Median & IQR \\
\hline Quantity (g) & 715 & 448,1198 & $38 \cdot 3$ & 2216 & 1758,2758 \\
\hline Energy (kJ) & 2578 & 1925,3595 & 36.9 & 7650 & 6134,9234 \\
\hline Energy (kcal) & 616 & 310,859 & & 1828 & 1466, 2207 \\
\hline Protein (g) & $18 \cdot 4$ & $13 \cdot 0,26 \cdot 2$ & $31 \cdot 1$ & $68 \cdot 6$ & $52 \cdot 9,86 \cdot 9$ \\
\hline Carbohydrate (g) & 83.9 & $61 \cdot 9,116 \cdot 4$ & 39.5 & $229 \cdot 6$ & $185 \cdot 7,282 \cdot 2$ \\
\hline Total fat $(\mathrm{g})$ & $19 \cdot 4$ & $12 \cdot 4,32 \cdot 2$ & 35.5 & $64 \cdot 0$ & $47 \cdot 5,82 \cdot 8$ \\
\hline Saturated fat (g) & $8 \cdot 1$ & $4 \cdot 4,13 \cdot 2$ & $35 \cdot 0$ & $26 \cdot 0$ & $18 \cdot 3,35 \cdot 2$ \\
\hline Added sugars (g) & 11.9 & $4 \cdot 3,26 \cdot 4$ & $36 \cdot 4$ & 44.9 & $25 \cdot 1,75 \cdot 6$ \\
\hline Fibre $(\mathrm{g})$ & $7 \cdot 7$ & $5 \cdot 2,10 \cdot 4$ & $40 \cdot 9$ & $19 \cdot 8$ & $14 \cdot 7,25 \cdot 7$ \\
\hline $\mathrm{Ca}(\mathrm{mg})$ & $198 \cdot 3$ & $100 \cdot 4,353 \cdot 5$ & 32.9 & 739.9 & $505 \cdot 0,1061.5$ \\
\hline $\mathrm{Na}(\mathrm{mg})$ & 830 & 589,1160 & 42.9 & 2128 & 1577, 2832 \\
\hline Vitamin C (mg) & $15 \cdot 6$ & $65 \cdot 2,1758 \cdot 7$ & 38.4 & $65 \cdot 2$ & $33 \cdot 4,122 \cdot 7$ \\
\hline Iron (mg) & $2 \cdot 6$ & $1 \cdot 8,3 \cdot 6$ & $32 \cdot 0$ & $9 \cdot 3$ & $6 \cdot 9,12 \cdot 1$ \\
\hline
\end{tabular}

IQR, interquartile range.

*Percentage contribution calculated for each participant and used to calculate mean.

Table 3 Median serves of Australian Dietary Guideline food groups consumed by 5 - to 12-year-old children during school hours and across the total day using the NNPAS 2011-2012 ( $n$ 795)

\begin{tabular}{|c|c|c|c|c|}
\hline & \multicolumn{4}{|c|}{ Serves consumed } \\
\hline & \multicolumn{2}{|c|}{$\begin{array}{l}\text { Within } \\
\text { school }\end{array}$} & \multicolumn{2}{|c|}{ Total day } \\
\hline & Median & IQR & Median & IQR \\
\hline Grains and cereals & 2 & 1,2 & 4 & 3,6 \\
\hline Vegetables and legumes & 0 & 0,1 & 1 & 0,3 \\
\hline Fruit & 1 & 0,1 & 1 & 1,3 \\
\hline $\begin{array}{l}\text { Milk, yoghurt, cheese and/or alter- } \\
\text { natives }\end{array}$ & 0 & 0,1 & 1 & 1,2 \\
\hline $\begin{array}{l}\text { Lean meat and poultry, fish, eggs, } \\
\text { nuts and seeds, and legumes/ } \\
\text { beans }\end{array}$ & 0 & 0,0 & 1 & 0,2 \\
\hline Discretionary choices & 2 & 1,3 & 5 & 3,7 \\
\hline
\end{tabular}

IQR, interquartile range.

For primary-aged children, recommendations range from 4 to 6 grain and cereal serves, 4.5 to 5.5 vegetable and legume serves, 1.5 to 2 fruit serves, 1.5 to 3.5 serves of milk, yoghurt, cheese and/or alternatives and 1.5 to 2.5 serves of lean meat and poultry, fish, eggs, nuts and seeds, and legumes/beans across the total day ${ }^{(1)}$.

It is recommended that more active children or adolescents have between 0 and 3 addition serves from the five food groups or discretionary choices ${ }^{(1)}$.

indicating older children may consume more discretionary choices.

\section{Sensitivity analyses}

Sensitivity analyses were completed for the conservative school days population, of 560 primary aged children (see online Supplemental Table S3 and S4). The population of conservative dates had fewer participants who were affected by overweight or obesity (17.9\%), compared with the primary sample (19.7\%). Energy and nutrients analyses showed a similar pattern of results, with values marginally lower during school hours for the conservative sample.
Median energy intake during school hours was $2472 \mathrm{~kJ}$ (IQR 1884, $3308 \mathrm{~kJ}$ ) contributing 34.7\% to total day energy intake, compared to the primary sample median of $2578 \mathrm{~kJ}$ (IQR 1925, $3595 \mathrm{~kJ}$ ), contributing 36.9\%. To determine if this variance was consistent, the median values for intake of Australian Dietary Guideline food groups were calculated, with the results almost identical between the two samples.

\section{Discussion}

This study profiled 5- to 12-year-old children's dietary intake during school hours, and contribution to total day intake, using a nationally representative sample of Australian children. Children are consuming approximately one-third of their daily energy intake during school hours, with a considerable proportion ( $44 \%$ ) of this being sourced from discretionary choices. Many children are consuming fruit and grain serves during school hours yet are underconsuming other food groups, such as vegetables, dairy and alternatives and meat and alternatives. Therefore, contributing to few children meeting Australian Dietary Guideline recommendations for the total day. These results reinforce schools as a key nutrition promotion setting and provide direction to inform intervention efforts in the future.

The current study finding that food consumed during school hours provides $37 \%$ of children's daily energy intake is consistent with previous findings, with 1995 data showing school food contributed $37 \%$ of daily intake ${ }^{(16)}$. There is a noted discrepancy in the energy intake in comparison with previous findings, with an overall $600 \mathrm{~kJ}$ increase in food consumed across the total day in the 2011-2012 survey data, compared to the 1995 National Nutrition Survey ${ }^{(16)}$. Despite children's diets becoming 
The dietary intake of children during school

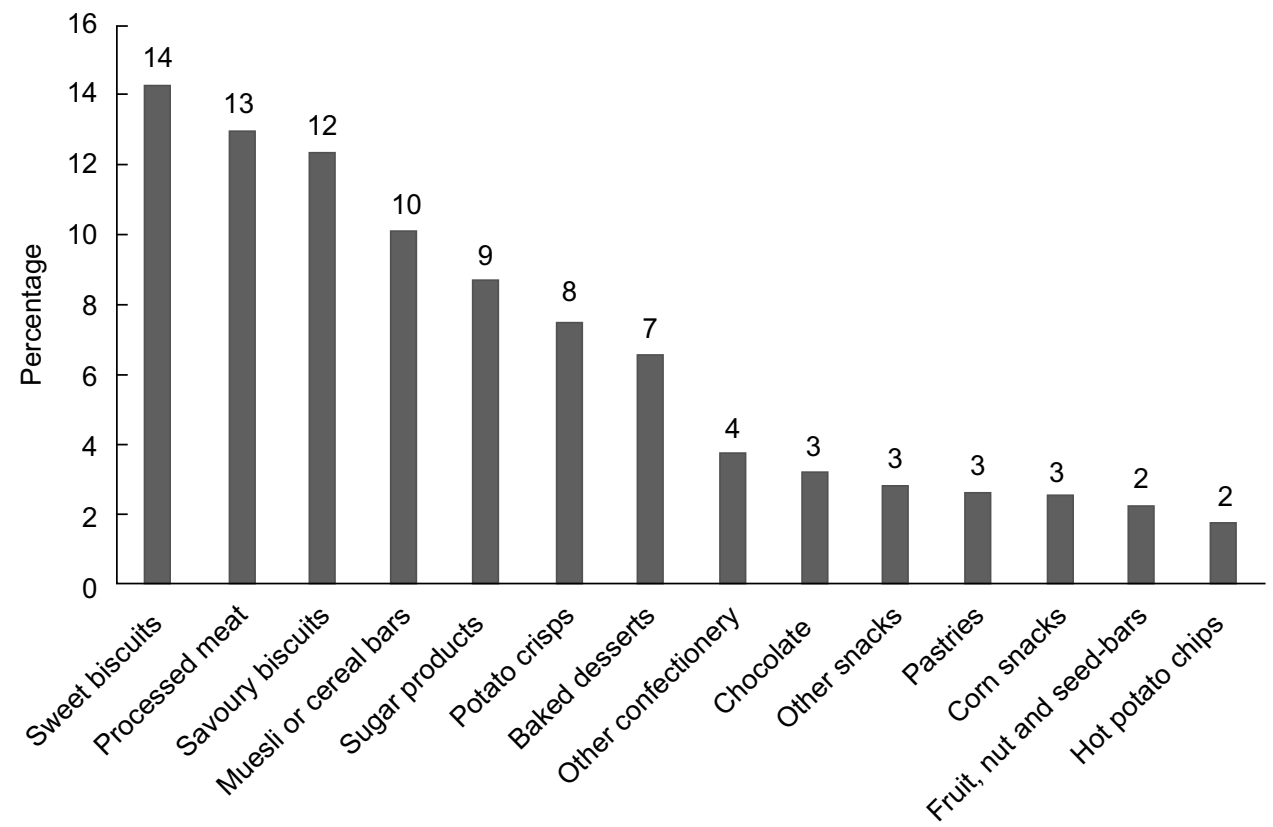

Fig. 2 Most frequently consumed food product categories* of discretionary foods during school hours by Australian children aged between 5- and- 12-years using the NNPAS 2011-2012.

*Percentage of discretionary food products consumed within school in each food sub-category, out 1537 discretionary food products

higher in energy overall, the energy consumed during school remained consistent over the decade between national surveys (1995 $2602 \mathrm{~kJ}$ v. 2011/12 $2578 \mathrm{~kJ})^{(16)}$. Our findings that $44 \%$ of children's energy during school hours were from discretionary choices is of concern and consistent with previous findings in Australian samples $^{(2,22)}$. A 2019 study examining food provided in lunchboxes to catholic school children found discretionary choices contributed $38.8 \%$ of the energy provided in lunchboxes ${ }^{(22)}$. This finding also aligns with evidence of children's intake across the total day, showing children aged 2 to 18 years are consistently over-consuming discretionary choices, with these products providing approximately $40 \%$ of daily energy intake, regardless of the school or home environment ${ }^{(2)}$. Discretionary choices products may be displacing products from the five food groups and contributing to poor consumption of these essential food groups during school hours ${ }^{(35)}$.

The most consumed food products during school hours included bread, fruit and discretionary choices, such as biscuits and snack items, which are highly marketed lunchbox products ${ }^{(36)}$ and are convenient with high child acceptability. The consumption patterns of these foods were consistent with previous Australian findings ${ }^{(16)}$, showing that between the 1995 and 2011-2012 national surveys there have been no notable improvements in the nutritional profile of school lunchboxes. The high intake of bread, fruit and discretionary choices is also consistently observed in more recent studies within smaller jurisdictions within Australian $^{(22-24)}$, and international evidence ${ }^{(10,12)}$. A study investigating intake of South Australian primary schoolaged children (8-11 years) in 2010 showed that children were consuming fruit or discretionary choices during morning snack break, and sandwiches during the lunch break $^{(12)}$. Further evidence has shown that over half of children's total day intake of discretionary choices occurs during snack eating occasions ${ }^{(37)}$, which corresponds with common morning snack breaks in Australian schools. To address commonly consumed discretionary choices, school-based interventions may consider targeting snack eating occasions in school, to encourage vegetable snack consumption $^{(21)}$, and discourage high sugar, fat and/or $\mathrm{Na}$ food and beverages.

The lack of notable change in school dietary intake of Australian children over time suggests that existing nutrition guidelines and interventions in schools have had little effect on improving the diet quality of primary-aged children. In addition, there has been limited implementation of recommended international guidelines, with no national guidelines in place supporting the provision of healthy foods at school via lunchboxes. There are numerous factors that may have limited a change in children's school food dietary intake, if not addressed in previous interventions. Previous research in Australia has highlighted the many barriers' families and canteen services face when packing, preparing and providing lunches to children in school hours, such as child preferences, time, cost and food safety ${ }^{(20,38)}$. The poor consumption of vegetables, milk and alternatives, and meat and alternatives food groups in addition to high intake of discretionary choices may 
be a reflection of the Australian school food model, including the limited facilities available, for both lunchbox and canteen provided foods. These intake patterns align with the barriers expressed by parents about food safety. However, it is noted that high discretionary choices intake patterns are not solely experienced in lunchbox food provision models. For example, in London, England, where food is prepared and provided by the school setting, children's lunch intake is characterised by high intake of discretionary choices ${ }^{(12)}$.

Analyses showing no significant associations with sociodemographic characteristics and energy sourced from discretionary choices indicates poor diet quality during school hours is consistent across all children, irrespective of socioeconomic position. In comparison, a review of diet quality and socio-economic position has found notable differences in diet quality of children of varying family socio-economic status when considering intake across the whole day ${ }^{(39)}$. Furthermore, evidence using socio-economic indices for areas in NSW examining lunchbox provision has shown no associations during school hours ${ }^{(22)}$. This is consistent with international findings that school hour dietary quality was similar across socio-demographic groups ${ }^{(40)}$. The findings of this study suggest that school interventions should target children in schools across the population, regardless of socio-economic areas, to improve the diets of all Australian children during school hours.

Consistently, poor diet quality of primary-aged children during school hours suggests that new approaches to improve children's school food intake are warranted, including consideration of new policies, programmes, or alternate food provision models. One avenue may be to reimagine the school food system to meaningfully improve the nutrition at school, with our recent research consulting key stakeholders indicating a school lunch prepared onsite is a food provision model worth exploring in Australia ${ }^{(38)}$. A school-provided lunch model in Australia, has the potential to improve diet quality of school children, if the appropriate processes and systems are put in place to ensure lunches provided align with national dietary guidelines. Potential benefits of a school-provided lunch model are evidenced by school-provided lunch models in other countries which allow for greater vegetable intake and provision ${ }^{(12-14)}$. The current study provides important insight into Australian children's intake at school to direct future research to continue exploring policies, programmes or alternative approaches to meaningfully change school food patterns. However, continued comprehensive analyses are needed to better understand trends and diet patterns of school children.

Key strengths of this secondary analysis include the use of dietary intake data of a national sample, collected via the validated multiple automated pass method ${ }^{(25)}$. Limitations include that while the NNPAS 2011-2012 is the latest available national dietary intake data it was collected 10 years ago and may not reflect current dietary patterns and any potential changes to children's intake due to school nutrition promotion initiatives since this data collection ${ }^{(19,21)}$. As a result, continued analyses are warranted to provide updated Australian evidence to better understand trends and diet patterns of school children. Therefore, repeating these comprehensive analyses to understand both the nutrients and foods consumed by children with ongoing national survey data is recommended. Other limitations of this study relate to the variables accessible for this secondary analysis. State or territory of residence and postcode data was not available in the dataset to ensure anonymity of participants. Lack of these variables resulted in the potential for recalls completed on school holiday or public holiday dates for certain states/territories being included in the primary analysis sample, as well as preventing comparisons in intake to be made across areas of Australia. However, completing sensitivity analyses of the conservative school dates sample, which revealed consistent patterns in the results suggested incidental inclusion of non-school day recalls had limited impact. In addition, the data did not differentiate whether food were packed from home or purchased from school canteens, hence cannot provide guidance to tailor future interventions towards specific modes of food provision. Finally, this analysis only used a single day of intake, hence may not reflect usual intake of participants.

\section{Conclusions}

The present study provides an update and extension of previous research to comprehensively describe Australian children's dietary intake during school hours, using a national sample. Australian primary-aged children were found to consume a third (37\%) of their daily energy intake during school hours, with $44 \%$ of that energy intake being sourced from discretionary choices. Commonly consumed discretionary food products during school hours include sweet and savoury biscuits, processed meats, and muesliand cereal bars. Current findings are consistent with previous Australian evidence over 17 years, which calls for considering new approaches to tackling school food intake, including exploring alternate policy approaches, programmes and school food provision models to meaningfully improve primary-aged children's intake during school hours.

\section{Acknowledgements}

Acknowledgements: None. Financial support: RS is supported by a NHMRC MRFF Investigator Fellowship (1194768). DZ is supported by a NHMRC Centre for Research Excellence in Early Prevention of Obesity in Childhood (1101675). Conflict of interest: There are no 
conflicts of interest. Authorship: A.C.M., B.J.J., D.Z. and R.K.G. were involved in project conceptualisation. A.C.M. designed and conducted the research, analysed data and drafted the manuscript. B.J.J., D.Z. and R.K.G. provided academic supervision and support for the work. All authors interpreted the results, contributed to, read and approved the final manuscript. Ethics of human subject participation: Ethics approval was not required as all data were provided by the Australian Bureau of Statistics in deidentified format.

\section{Supplementary material}

For supplementary material accompanying this paper visit https://doi.org/10.1017/S1368980021003888

\section{References}

1. National Health and Medical Research Council (2013) Eat for Health: Educator Guide. Canberra, ACT: National Health and Medical Research Council.

2. Johnson BJ, Bell LK, Zarnowiecki D et al. (2017) Contribution of discretionary foods and drinks to Australian children's intake of energy, saturated fat, added sugars and salt. Children 4, 104-118.

3. Holmes KL, Rollo ME \& Collins CE (2018) Do the contemporary dietary patterns of children align with national food and nutrient recommendations? J Hum Nutr Diet 31, 670-682.

4. Andersen R, Biltoft-Jensen A, Christensen T et al. (2015) What do Danish children eat, and does the diet meet the recommendations? Baseline data from the OPUS school meal study. J Nutr Sci $\mathbf{4}$, e29.

5. Krebs-Smith SM, Guenther PM, Subar AF et al. (2010) Americans do not meet federal dietary recommendations. J Nutr 140, 1832-1838.

6. World Health Organisation (2020) Health Promoting Schools. https://www.who.int/health-topics/healthpromoting-schools\#tab=tab_3 (accessed August 2020).

7. UNICEF (2019) Primary Education. http://data.unicef.org/ topic/education/primary-education/ (accessed November 2020).

8. Colombo PE, Patterson E, Elinder LS et al. (2020) The importance of school lunches to the overall dietary intake of children in Sweden: a nationally representative study. Public Health Nutr 23, 1705-1715.

9. Tugault-Lafleur CN, Black JL \& Barr SI (2017) Examining school-day dietary intakes among Canadian children. Appl Physiol Nutr Metab 42, 1064-1072.

10. Harrison F, Jennings A, Jones A et al. (2013) Food and drink consumption at school lunchtime: the impact of lunch type and contribution to overall intake in British 9-10-year-old children. Public Health Nutr 16, 1132-1139.

11. Osowski CP, Lindroos AK, Barbieri HE et al. (2015) The contribution of school meals to energy and nutrient intake of Swedish children in relation to dietary guidelines. Food Nutr Res 59, 27563.

12. Zarnowiecki D, Christian MS, Dollman J et al. (2018) Comparison of school day eating behaviours of 8-11 year old children from Adelaide, South Australia, and London, England. AIMS Public Health 5, 394-410.

13. Upton D, Upton P \& Taylor C (2012) Fruit and vegetable intake of primary school children: a study of school meals. J Hum Nutr Diet 25, 557-562.
14. Huang ZR, Gao RY, Bawuerjiang N et al. (2017) Food and nutrients intake in the school lunch program among school children in Shanghai, China. Nutrients 9, 582.

15. Walton J, Hannon EM \& Flynn A (2015) Nutritional quality of the school-day diet in Irish children (5-12 years). J Hum Nutr Diet 28, 73-82.

16. Bell AC \& Swinburn BA (2004) What are the key food groups to target for preventing obesity and improving nutrition in schools? Eur J Clin Nutr 58, 258-263.

17. Lucas PJ, Patterson E, Sacks G et al. (2017) Preschool and school meal policies: an overview of what we know about regulation, implementation, and impact on diet in the UK, Sweden, and Australia. Nutrients 9, 736-756.

18. World Health Organization (2008) School Policy Framework: Implementation of the WHO Global Strategy on Diet, Physical Activity and Health. Geneva: World Health Organization.

19. The Australian Department of Health (2013) 2010 National Healthy School Canteens Guidelines. https://www1.health. gov.au/internet/main/publishing.nsf/Content/phd-nutritioncanteens (accessed August 2020).

20. Bathgate K \& Begley A (2011) 'It's very hard to find what to put in the kid's lunch': what Perth parents think about food for school lunch boxes. Nutr Diet 68, 21-26.

21. Myers G, Wright S, Blane S et al. (2018) A process and outcome evaluation of an in-class vegetable promotion program. Appetite 125, 182-189.

22. Sutherland R, Nathan N, Brown A et al. (2020) A crosssectional study to determine the energy density and nutritional quality of primary-school children's lunchboxes. Public Health Nutr 23, 1108-1116.

23. Grimes CA, Riddell LJ \& Nowson CA (2014) Nutrient and core and non-core food intake of Australian schoolchildren differs on school days compared to non-school days. Appetite 83, 104-111.

24. Sanigorski AM, Bell AC, Kremer PJ et al. (2005) Lunchbox contents of Australian school children: room for improvement. Eur J Clin Nutr 59, 1310-1316.

25. Australian Bureau of Statistics (2017) 4363.0.55.001 Australian Health Survey: Users' Guide, 2011-2013. Australian Health Survey. https://www.abs.gov.au/ausstats/ abs@.nsf/Latestproducts/4363.0.55.001Main\%20Features120 11-13?opendocument\&tabname $=$ Summary $\&$ prodno $=4363.0$. $55.001 \&$ issue $=2011-13 \&$ num $=\& v i e w=($ accessed July 2020$)$.

26. Steinfeldt L, Anand J \& Murayi T (2013) Food reporting patterns in the USDA automated multiple-pass method. Procedia Food Sci 2, 145-156.

27. Black AE, Goldberg GR, Jebb SA et al. (1991) Critical evaluation of energy intake data using fundamental principles of energy physiology: 2. Evaluating the results of published surveys. Eur J Clin Nutr 45, 583-599.

28. Goldberg GR, Black AE, Jebb SA et al. (1991) Critical evaluation of energy intake data using fundamental principles of energy physiology: 1. Derivation of cut-off limits to identify under-recording. Eur J Clin Nutr 45, 569-581.

29. Rangan A, Allman-Farinelli M, Donohoe E et al. (2014) Misreporting of energy intake in the 2007 Australian children's survey: differences in the reporting of food types between plausible, under- and over-reporters of energy intake. J Hum Nutr Diet 27, 450-458.

30. Food Standards Australia and New Zealand (2019) AUSNUT 2011-2013. https://www.foodstandards.gov.au/science/mo nitoringnutrients/ausnut/Pages/about.aspx (accessed July 2020).

31. Cole TJ, Bellizzi MC, Flegal KM et al. (2000) Establishing a standard definition for child overweight and obesity worldwide: international survey. BMJ 320, 1240-1243.

32. Thomson JL, Tussing-Humphreys LM, Goodman MH et al. (2018) Diet quality in a nationally representative sample of American children by sociodemographic characteristics. Am J Clin Nutr 109, 127-138. 
33. Thivel D, Aucouturier J, Doucet É et al. (2013) Daily energy balance in children and adolescents. Does energy expenditure predict subsequent energy intake? Appetite 60, 58-64.

34. Torra V, Domingo-Ferrer J, Mateo-Sanz JM et al. (2006) Regression for ordinal variables without underlying continuous variables. Inf Sci 176, 465-474.

35. Bowman SA, Gortmaker SL, Ebbeling CB et al. (2004) Effects of fast-food consumption on energy intake and diet quality among children in a national household survey. Pediatrics $\mathbf{1 1 3}, 112$.

36. Elliott C (2019) Tracking kids' food: comparing the nutritional value and marketing appeals of child-targeted supermarket products over time. Nutrients 11, 1850.
37. Rebuli MA, Williams G, James-Martin G et al. (2020) Food group intake at self-reported eating occasions across the day: secondary analysis of the Australian national nutrition survey 2011-2012. Public Health Nutr 23, 1-14.

38. Johnson BJ, Zarnowiecki D, Hutchinson CL et al. (2020) Stakeholder generated ideas for alternative school food provision models in Australia using the nominal group technique. Int J Environ Res Public Health 17, 7935.

39. Darmon N \& Drewnowski A (2008) Does social class predict diet quality? Am J Clin Nutr 87, 1107-1117.

40. Tugault-Lafleur CN, Barr SI \& Black JL (2019) Examining differences in school hour and school day dietary quality among Canadian children between 2004 and 2015. Public Health Nutr 22, 3051-3062. 